\title{
La lucha contra la desinformación sobre la COVID-19 en Brasil: estudio exploratorio de las agencias de verificación Fato ou Fake y Lupa
}

\author{
Allysson Martins \\ Universidade Federal de Rondônia \\ allyssonviana@unir.br \\ https://orcid.org/0000-0001-7805-6512
}

\author{
JuLIANA TEIXEIRA \\ Universidade Federal do Piauí \\ teixeira.juliana.rj@gmail.com \\ https://orcid.org/0000-0002-0631-7194
}

\author{
Ainara LaRRONDO \\ Universidad del País Vasco/ \\ Euskal Herriko Unibertsitatea \\ ainara.larrondo@ehu.eus \\ https://orcid.org/0000-0003-3303-4330
}

\section{The struggle against the desinformation on COVID-19 in Brazil: an exploratory study of the fact-checking agencies Fato ou Fake and Lupa}

\begin{abstract}
RESUMEN
Las redes sociales digitales no solo propagan contenido útil, sino que también contribuyen a difundir informaciones falsas que contribuyen a la desinformación de la sociedad en diferentes contextos geográficos, políticos y culturales. Las agencias digitales de verificación de hechos

-fact-checking en la terminología anglosajona- son vistas como un remedio eficaz contra este problema, junto con otras posibilidades o estrategias, como la exclusión de contenidos y la desmonetización de los propagadores.

En consideración al impacto de la pandemia del COVID-19 (coronavirus disease 2019) en Brasil, este artículo investiga la importancia dada por las agencias de fact-checking de ese país, Fato ou Fake y Lupa, a sus verificaciones de noticias falsas sobre la COVID-19. Si durante los períodos iniciales de propagación de la enfermedad en enero y febrero de 2020 , las verificaciones no alcanzaron el $20 \%$ del material informativo generado, a partir de marzo, las comprobaciones se intensificaron en relación a la pandemia, alcanzando una media de aproximadamente dos publicaciones por día.
\end{abstract}

ALABRAS CLAVE

Desinformación; Informaciones falsas; Agencias de verificación de hechos; Verificación periodística; COVID-19.

\section{ABSTRACT}

Digital social networks not only spread useful content, but also contribute to spreading false information that contributes to the misinformation of society in different geographical, political and cultural contexts. Digital fact-checking agencies are seen as an effective remedy against this problem, along with other possibilities or strategies, such as the exclusion of content and the demonetization of propagators. In consideration of the impact of the COVID-19 (coronavirus disease 2019) pandemic in Brazil, this article investigates the importance given by the fact-checking agencies of that country, Fato ou Fake and Lupa, to their verification of false news on the COVID-19. If during the initial periods of spread of the disease in January and February 2020 , the verifications did not reach $20 \%$ of the information material generated, as of March, the verifications were intensified in relation to the pandemic, reaching an average of approximately two posts per day.

KEYWORDS

Misinformation; Fake news; Fact-checking agencies; Journalistic investigation; COVID-19. 


\section{Introducción}

La propagación mediática de los contenidos digitales basada en la lógica de una cultura de conexión (Jenkins, 2014) se intensifica por las posibilidades de interacción y participación del consumidor en Internet. A medida que aumenta el número de dispositivos portátiles de fácil manejo, se mejora la producción y circulación de contenidos en todos los formatos y formas. Estos dispositivos conectados en movilidad a través de redes digitales e inalámbricas, además de capturar información, permiten la publicación en tiempo real y en entornos de amplia circulación (Lemos, 2004; Briggs, 2013; Canavilhas; Satuf, 2014; Silva, 2015).

En este contexto, la asimilación del uso de las redes sociales en la vida cotidiana contribuye a una difusión de la información de manera totalmente espontánea, motiva en muchos casos por valores como la espectacularización, la emocionalización o la personalización.

Esa menor dependencia del intermediario significó que los políticos y otras figuras públicas pudieran relacionarse directamente con los individuos, teniendo suficiente control sobre los medios de producción y circulación de la información, como los perfiles en las redes sociales. "En este sentido, el campo periodístico ya no es la instancia exclusiva de producción y mediación de eventos" (Borcezi; Morais, 2019, p. 72). Además de estos dos aspectos -menos exclusividad y descrédito-, las fake news encontraron terreno fértil porque el periodismo aumenta su fetiche por la velocidad (Moretzsohn, 2002), afectando, especialmente, la verificación, considerada la primera etapa de la rutina productiva.

Precisamente, la relación más estrecha entre consumidores y productores hace que el contenido se ajuste a las expectativas, intereses y necesidades de quienes circulan y propagan productos digitales casi ininterrumpidamente. Con las redes sociales digitales, Zago $(2010,2011)$ incluso propone un nuevo modelo de (re)circulación de contenido periodístico. Este panorama ha favorecido la propagación de contenido útil, pero también de informaciones de escasa calidad periodística e, incluso, informaciones no veraces. Desde la segunda mitad de la década de 2010 es posible observar un proceso desarrollado y estructurado de desinformación a través de la difusión de información falsa en los medios digitales, distinguidas bajo el anglicismo fake news o la expresión general de "noticias falsas". Es cierto que el mismo término fake news ha sido cuestionado académica y científicamente en la medida en que las noticias (news) traen consigo la suposición de verdad y, por lo tanto, no pueden ser falsas (fakes).

En este contexto, el objetivo general de la investigación que encierra este artículo es estudiar los procedimientos aplicados en la lucha contra la información falsa en Brasil ante el COVID19. Concretamente, el objetivo principal es dar respuesta a dos cuestiones: ¿cuáles son los estándares de fake news que se propagan en el proceso de desinformación sobre COVID-19 en Brasil? y ¿cómo evalúan los sitios web de las agencias de verificación brasileñas esta información falsa y presentan los resultados al público? En este texto, presentamos los resultados exploratorios de la etapa inicial de esta doble pregunta, a través de la frecuencia de las verificaciones de la pandemia en los sitios web de las agencias de fact-checking Fato ou Fake y Lupa.

Los resultados forman parte de un análisis más amplio llevado a cabo entre enero y junio de 2020 sobre la cobertura mediática de la pandemia en los medios de comunicación brasileños, teniendo en cuenta que Brasil ha sido uno de los países más azotados por esta pandemia en todo el mundo. A partir de una revisión bibliográfica sobre fake news, el artículo discute temas como la credibilidad periodística y la innovación presente en las agencias digitales de fact-checking. La metodología de estudio proporciona una combinación del framing (Entman, 1993) con el análisis de contenido (Bardin, 2004), como se detallará más adelante. Se evalúa así el framing de medios en el proceso de desinformación relacionado con la COVID-19, además del modo en el que las estrategias de las agencias de verificación confirman o refutan este framing "general". En otras palabras, identificamos las innovaciones aplicadas por estas agencias para combatir la información falsa sobre la pandemia en Brasil.

\section{Un término aún es impreciso: ¿una estrategia útil?}

La imprecisión común del término fake news se debe, entre otros factores, a la multiplicidad de aspectos considerados en su análisis, relacionados con seis dimensiones: sátira -humor basado en hechos; parodia -humor con creación de noticias; fabricación -invención de información, manipulación; edición o descontextualización de formatos de medios, facilitado por el aspecto digital; publicidad -contenido pagado en formato periodístico; y publicidad: personas e instituciones remuneradas para guiar la opinión pública sin transparencia (Tandoc Jr., Lim; Ling, 2017).

Aunque la imprecisión común persiste a la hora de definir las fake news, es posible reducir su definición a tres opciones: (1) errores no intencionales, lo que resulta en la publicación de información falsa; (2) parodia del periodismo, con sátiras que aportan información falsa (o verdadera con exagero) en la estructura y en el formato periodístico; y (3) mentiras deliberadas, con producción intencional para difundir información falsa, con poca observancia en el periodismo.

Los dos primeros casos se observan durante el siglo pasado, con la idea de que las fake news aparecen por primera vez en 1925 en la Harper Magazine para tratar los errores de los profesionales de la prensa y el sensacionalismo que preva- 
leció en la prensa estadounidense en el siglo XIX (Schudson, 2008; Schudson; Zelizer, 2017). Todavía así, en el periodismo, "el objetivo nunca será engañar a los lectores, lo que nos hace querer separar las false news (periodismo) de las fake news (manipulación y desinformación)" (Meneses, 2018: 42). A finales del siglo $X X$, los estudios comenzaron a dar cuenta de producciones humorísticas que parodiaban el periodismo, algo que existe incluso hoy en día. La intención aquí tampoco es el engaño, sino la diversión, como es posible observar en algunos programas de la televisión brasileña de Casseta \& Planeta, ¡Urgente!, Furo MTV y Fora de Hora, además de diarios como The Piauí Herald, Sensacionalista, Diário de Barrelas, 0 Bairrista, G17 y Diário Pernambucano.

Las tres dimensiones señaladas comprenden un ecosistema heterogéneo de información falsa, aunque solo la última tiene, de hecho, propósitos de desinformación. Como estas tres dimensiones se rigen por reglas y significados totalmente diferentes, el uso actual de la expresión fake news se vincula mayoritariamente a la tercera opción -mentiras deliberadasdistanciándose de las otras dos posibilidades ya existentes antes de la popularización de Internet, distinguidas también en ocasiones como false news. Mientras que "fake" se refiere al fraude (desinformación), "false" está así más cerca del concepto de incorrecto (misinformation).

La elección presidencial de Estados Unidos en el año 2016, sustentada en la disputa electoral entre los candidatos Donald Trump y Hillary Clinton, popularizó el uso del término fake news. Mientras Clinton se refirió a este proceso contemporáneo de desinformación, Trump trató de criminalizar la información periodística desfavorable o, a lo sumo, la que contenía algún error (false news). Esta estrategia de Trump de desarrollar desconfianza e incredulidad en una institución como la periodística habría contribuido a generar un escenario favorable para el proceso de desinformación a través de las fake news (Meneses, 2018; Tandoc Jr., Lim; Ling, 2017).

Por fake news, por lo tanto, cabe entender un fenómeno contemporáneo de manipulación de información falsa presentada como real y creíble dentro de la lógica de difusión de los medios con el objetivo de desinformar, especialmente en medios digitales sociales, que pueden o no imitar la estructura y formato periodístico. Ese mimetismo sirve para confundir a quienes reciben esta información falsa y dar mayor credibilidad a ese contenido, facilitando la desinformación y su difusión (Meneses, 2018; Tandoc Jr., Lim; Ling, 2017).

\section{Fake news en el contexto digital y periodístico}

Históricamente las luchas de poder, las guerras y las campañas políticas han contribuido a la difusión de información falsa con miras a la desinformación deliberada y planificada. Los tiempos difíciles y de agitación social, como los generados por la pandemia del COVID-19, resultan también muy favorables para la propagación de desinformaciones intencionadas. Otra evidencia demostrada es que la Web contribuye a desarrollar un proceso de difusión de información falsa con proporciones sin precedentes. Entre otros motivos, los medios digitales facilitan la producción informativa, hacen que la publicación sea más barata y rápida, permiten una mayor edición y manipulación, aumentan el flujo y automatizan la difusión. En estos medios parece haber una cierta relajación de los límites entre los periodistas y otros productores de contenido.

El flujo comunicacional con los medios digitales alcanza diferentes proporciones que los medios de comunicación tradicionales; ahora, es más amplio y heterogéneo, además de incluir una aplicación de mensajería privada. Mientras que Facebook, con más de 127 millones de usuarios mensuales en Brasil, permite el escrutinio público de sus mensajes, WhatsApp, con más de 120 millones de seguidores mensuales, permite el intercambio privado, lo que hace que sea más difícil combatir esa desinformación, más aún cuando es emitida o propagada por personas de confianza, con las que se tiene algún tipo de conexión.

En este período, cuando la posibilidad de producir y distribuir información ya no es exclusiva para periodistas y medios, y cuando aumenta la desacreditación del periodismo, autores como Spinelli y Santos (2018) reivindican la idoneidad del momento para reforzar la credibilidad de la profesión, renovando este atributo con técnicas modernas de verificación (Eichler; Kalsing; Gruszynski, 2018; Gruszynski et al., 2020).

En tiempos de fake news, ha surgido incluso iniciativas para delimitar los indicadores de la credibilidad del periodismo. Tal sería el caso de Trust Project, un consorcio internacional de organizaciones periodísticas creado en 2014 con el objetivo de favorecer a los productores de contenido con un compromiso ético. Las organizaciones del citado consorcio se comprometen a emplear estándares para mantener o expandir la confianza pública en el periodismo (Träsel; Lisboa; Reis, 2018). Especialmente porque, para ser válidas, las fake news necesitan que la gente crea que lo "fake" es real o, al menos, creíble.

La lucha contra las fake news en Internet, especialmente en redes sociales, se ha convertido en un objetivo prioritario, ante la confusión y el daño ético que pueden generar. Además de medidas como la creación de leyes y de mecanismos para la notificación de contenidos falsos por parte de las audiencias, hasta la fecha el problema también se ha abordado desde la verificación constante de la información, llevada a cabo por agencias de fact-checking. Éstas proliferan en entornos digitales y sitios web vinculados a empresas periodísticas tradicionales. En tiempos de descrédito y ataques constantes, por el contrario, no solo el trabajo tradicional de los periodis- 
tas adquiere relevancia, sino también el de las agencias que emplean técnicas periodísticas.

Las agencias de verificación pretenden buscar la verdad, aunque a través de los propios rituales periodísticos, como el uso y presentación de sus fuentes, especialmente las documentales, presentes, por ejemplo, en hasta 2/3 de los cheques evaluados por Fernandes, Oliveira y Gomes (2019). Para Alencar e Dourado (2020) y Santos y Maurer (2020), el periodismo tiene una excesiva dependencia de las fuentes, transmitiendo información que no puede verificar, sin embargo, son estas las que normalmente sirven de base a las agencias de control, que prueban que fue posible, eso sí, evalúe la veracidad o no del contenido.

El contexto descrito hasta ahora revela que se han verificado algunos cambios en el proceso de producción del periodismo en internet, aunque se perciben varias continuidades. Hoy en día seguimos hablando de etapas tradicionales del periodismo, pero en un contexto diferenciado, más acelerado, que requiere más destreza de quienes ejercen la función de periodista, solo que con menos tiempo disponible para aprender a ejercerlo. Esto significa que estamos ante un proceso de transición de transformaciones cuantitativas a cualitativas, que, sin embargo, aún no se han concretado como un salto, generando innovaciones expresivas.

\section{Iniciativas innovadoras de fact-checking para el periodismo}

Este artículo prioriza su interés no tanto en el impacto de las fake news en aquellos que interactúan con esta información inexacta, sino en el proceso de producción periodística acometido para combatirlas por parte de dos agencias de verificación de gran relevancia en Brasil, especialmente en relación con la etapa inicial de propagación de la pandemia (primer semestre del año 2020). Así, presentamos datos sobre la verificación de información sobre COVID-19. Partimos de la premisa de las agencias objeto de estudio innovan en el contexto digital actual cuando buscan agregar calidad a su contenido periodístico, en función de una garantía de credibilidad de sus noticias. Más adelante, abordaremos los problemas para acometer esta innovación. No en vano, según destacan Palacios et al. (2015), se trata de un proceso difícil de definir y cuantificar, debido a la gran y compleja variedad de tipologías, indicadores y escalas de medición.

Vale la pena mencionar que los sitios web de las agencias de verificación de hechos comenzaron en la década de 2000, con factcheck.org, y PolitiFact, ganadora de un Premio Pulitzer en el año 2009. Un día después del Día de los Inocentes, se celebra el Día Internacional de Fact-checking, que tiene una red internacional, la International Fact-checking Netword (IFCN), con códigos de conducta para sus organizaciones afiliadas, como la ausencia de partidismo o la transparencia de las fuentes. Estas agencias iniciaron su andadura evaluando el discurso de políticos y figuras públicas, y hoy se presentan como una "vacuna" contra fake news, sumándose a otras estrategias de combate, como la exclusión de contenidos y la desmonetización de los propagadores por parte de compañías como Facebook y Google (Eichler; Kalsing; Gruszynski, 2018; Gruszynski et al., 2020; Silva; Melo, 2020; Spinelli; Santos, 2018). Para una mayor efectividad, estas confrontaciones deberían combinarse con la alfabetización mediática (Spinelli; Santos, 2018), un procedimiento que, a la larga, debería preparar a las personas para reconocer la desinformación y, con ello, reducir el impacto de la información falsa en la sociedad.

Existen muchas agencias digitales de verificación de hechos que operan en la escena brasileña, entre las cuales cabe destacar Fato ou Fake, Lupa, Projeto Comprova, Aos Fatos, E-Farsas y Boatos. Todas se presentan como iniciativas de fact-checking llevadas a cabo por periodistas, con un proceso complejo no solo porque involucran a varios profesionales, sino principalmente porque, como cada acto de comunicación, consiste en un intercambio entre dos instancias diferentes: la producción y la recepción. Según Charaudeau (2009), esta distinción permite abordar la información como algo que no corresponde solo a la intención del productor y del receptor, sino como resultado de una cointencionalidad que abarca los efectos previstos, posibles y producidos. La relación establecida entre producción y recepción determinaría tres lugares de relevancia: el lugar de producción, la instancia receptora y el texto como producto que, como tal, está sujeto a ciertas condiciones de construcción.

También es posible relacionar estas agencias con la tipología propuesta por Graves y Cherubini (2016) que diferencia los sitios de fact-checking en Europa entre "modelo de redacción" y "modelo NGO", este último realizado fuera del entorno mediático tradicional. En Brasil, podemos verificar que ambos modelos se están llevando a cabo, pero los dos estudiados en este artículo son parte del primer tipo.

Estas consideraciones revelan la idea de que el periodismo, también aquel que propaga fake news, es un proceso de construcción negociado (Tuchmann, 1978). No debemos ni podemos considerar el proceso de producción como uniforme, estático o inmutable, ya que ocurre a través de la interacción de periodistas con otras categorías de actores movilizados para determinar los hechos y su posterior transformación en un evento mediático (Sodré, 2009; Traquina, 2005). Además, la producción periodística varía mucho según el tipo de información que se pretende transmitir y siempre hay cambios en la técnica y los roles que desempeña cada periodista en el proceso de producción (Herreros, 2003).

Aunque el contexto descrito hasta ahora revela cambios en el proceso de producción del periodismo en Internet con respecto a los soportes anteriores, también es posible hablar de claras continuidades. El proceso rutinario de preparación de 
contenido periodístico (Sousa, 2000; Wolf, 2005), en general, sigue organizándose en torno a la verificación, producción y edición (Erbolato, 2006; Lage, 2009). Lo que cambia es la forma en que los periodistas llevan a cabo estos procesos. Las etapas tradicionales del periodismo se mantienen, pero en un contexto diferente y acelerado que requiere más habilidad por parte de quienes ejercen la función periodística. Esto significa que nos enfrentamos a un proceso de transición de transformaciones cuantitativas a cualitativas, que, sin embargo, aún no puede ser percibido como un salto sustentado en grandes innovaciones.

En esta era de transición mediática, como advierte Jenkins (2009), las instrucciones son imprecisas y los resultados son impredecibles, ya que están marcados por decisiones tácticas y consecuencias inesperadas, signos confusos e intereses en conflicto. En cualquier caso, la recuperación de la credibilidad por parte del periodismo requiere el desarrollo de prácticas renovadas y más efectivas, que exploren nuevas formas de verificar, producir, editar y circular contenidos noticiosos en la web (Larrondo, 2007). El alto flujo de comunicación también ha requerido, al menos de los profesionales, una mayor atención desde la primera etapa de este sistema, lo que hace que la verificación sea cada vez más relevante.

\subsection{La verificación como etapa fundamental para un periodismo innovador}

Es necesario comprender que la verificación no se limita solo a la captura de información, ya que esta recopilación debe someterse a una evaluación de veracidad, es decir, si, después de todo, esa información es válida, creíble. Para esto, el profesional generalmente realiza este proceso a través de todo tipo de fuentes, personales (testigos, expertos, etc.) y documentales. Herreros (2003) clasifica la verificación como la etapa más importante del proceso de producción, ya que será esta decisión la que condicionará todo el proceso.

Aunque reconocemos que la verificación no se basa solo en criterios periodísticos, sino que también depende de las posibilidades económicas, tecnológicas y humanas de cada organización mediática, se sabe que una investigación de calidad siempre da prioridad a la precisión de los hechos, así como a la calificación, idoneidad y diversificación de fuentes a través de múltiples técnicas periodísticas. Según Barbeiro y Lima (2002: 72), "el rigor en la verificación de los hechos es decisivo para la calidad". Virissimo (2009) argumenta que una investigación rigurosa tiene el potencial de generar periodismo calificado, capaz de reconocer la complejidad de la sociedad, de ofrecer información precisa, verificada y presentada de manera equitativa, y de exponer opiniones divergentes.

La verificación aparece, por lo tanto, como una condición sine qua non de la práctica y producción del periodismo. Para Ward (2006) esta primera etapa de captura o investigación adquiere gran importancia y puede ser subclasificada en dos procesos, el de identificación y el de obtención. Según Machado (2003), el ciberespacio, debido a sus especificidades, se configuró como un amplio y nuevo espacio para recopilar información periodística, además de alterar su proceso de producción, aunque, en el momento de su expansión como cuarta gran plataforma de medios, la verificación por Internet aún no era un lugar común. Desde entonces, ha habido una creciente demanda para el desarrollo de nuevas técnicas de investigación y recopilación informativa para los periodistas, especialmente después de la creación y apropiación de redes sociales digitales a mediados y finales de la década de 2000. Estas técnicas son fundamentales debido a la amplia existencia de fuentes de flujos comunicativos, creando desafíos sin precedentes para los profesionales de la comunicación. Este problema ya se observó incluso cuando por Internet no circulaba una cantidad de información tan ingente como la actual, cuando no tenía a las personas y las audiencias tan implicadas a través de las redes sociales.

Esto muestra que la verificación periodística no ha perdido, ni perderá, su relevancia en la Web. Por el contrario, según Stovall (2004), independientemente de los medios, la investigación continuará siendo una de las actividades periodísticas más importantes en el contexto digital.

Otro aspecto fundamental a considerar en este análisis es que el uso de la Web para la verificación periodística es ambivalente: el carácter potencialmente ilimitado del almacenamiento de información puede favorecer su pluralidad, pero también presenta la difícil tarea de distinguir los contenidos relevantes y verdaderos de los que no lo son (Virissimo, 2009).

En un entorno con tales especificidades, el ejercicio del periodismo de calidad depende, por lo tanto, del establecimiento de criterios capaces de garantizar la fiabilidad del sistema de verificación, así como del dominio de las técnicas apropiadas para seleccionar y clasificar información de disímil carácter, con valores y propósitos desiguales (Machado, 2003). Quinn (2005) refuerza que el desafío actual es filtrar lo que no queremos saber y las personas que no queremos escuchar, lo que coloca el procesamiento y la investigación de los hechos como una de las principales responsabilidades periodísticas.

Un ejemplo de este crecimiento y valoración de la investigación son los sitios web de las agencias de verificación de hechos antes mencionados, que se centran más en este primer paso rutinario inherente al proceso periodístico. Aunque producen y editan el contenido, para llegar a un público más amplio y explicar los entresijos de esa información falsa sobre el proceso de desinformación, el papel principal de estas agencias está en una investigación rigurosa. Sus consumidores, de hecho, quieren saber si esa información es verdadera o no, a pesar de que la mayoría de las agencias hacen que la presentación de estos contenidos sea más compleja, con etiquetas que no siempre aseguran la veracidad o falsedad completa del 


EMenu fol FATO OU FAKE $\quad$ Q Buscar

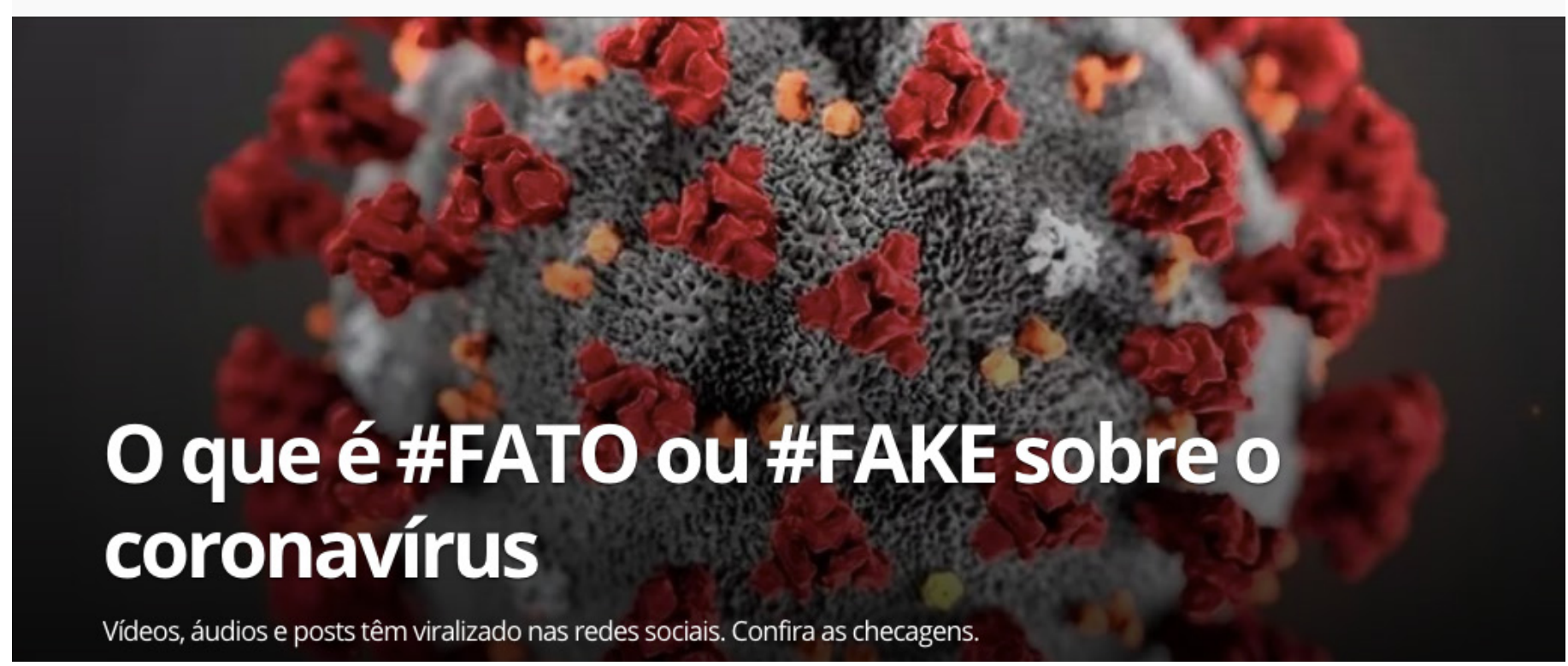

Figura 1. Verificación sobre COVID-19 destacada en la página principal de Fato ou Fake. Fuente: Portal G1.

contenido. Lupa, por ejemplo, usa nueve gradaciones de confiabilidad de la información, mientras que Fato ou Fake, por su parte, trabaja con el binarismo de su nombre (Verdad o Fake).

\section{Una exploración de la verificación de fake news sobre la COVID-19}

Los impactos de la desinformación van más allá de la confusión entre lo que es verdad o mentira, ya que pueden cambiar la conducta de las personas, culminando incluso en violencia física, como el caso emblemático del francotirador que invadió una pizzería porque creía que albergaba una red de pedofilia, una mentira propagada después de que el propietario apoyó a Hillary Clinton en la campaña presidencial de 2016. La difusión de fake news, cuando se trata de cuestiones relacionadas con la salud, como el movimiento contra la vacunación y tratamientos milagrosos, también conduce a la muerte, como en el caso de la fiebre amarilla en Brasil, cuando murieron más de 130 monos en menos de un mes, en el estado de Río de Janeiro. Esta propagación de la mentira se ve amplificada por el descrédito de otras instituciones más allá del periodismo, como la ciencia y la política o el Estado (Gruszynski et al., 2020; Teixeira; Costa, 2020).

Por lo que se refiere específicamente a las informaciones falsas sobre la salud, debe tenerse en cuenta que se propagan a través del miedo y de la preocupación, sin tener en cuenta que la acción individual tiene un impacto colectivo. "Al mismo tiempo que las fake news invaden las redes digitales en defensa de la vida, desprotegen al cuerpo de enfermedades y lo ponen en riesgo de muerte" (Teixeira; Costa, 2020: 79). Aunque el Programa Nacional de Inmunización de Brasil es reconocido internacionalmente, la desinformación ha provocado la reaparición de algunas enfermedades, como el sarampión, la poliomielitis y la fiebre amarilla. Este nuevo escenario requirió que el Ministerio de Salud creara en 2018 un canal directo para negar fake news, Saúde Sem Fake News. En 2020, desarrolló un hotsite con información oficial sobre COVID-19, obteniendo la mayor importancia en su página principal.

Las agencias brasileñas de fact-checking han adquirido un protagonismo muy relevante durante el período pandémico del nuevo coronavirus, COVID-19, cuyos síntomas principales son fiebre, tos y dificultad para respirar, que comienza en diciembre de 2019 en China. Mientras que la agencia Fato ou Fake organizó todas las producciones sobre COVID-19 en una nueva dirección (https://g1.globo.com/fato-ou-fake/coronavirus/), destacada en su página principal, Lupa permite la navegación en la verificación de esta información a través de cuatro categorías: \# coronavirus, \# novo-coronavirus, \#covid y \# covid-19 (Figura 1).

Estas dos agencias fueron seleccionadas como caso de estudio por su utilidad para ejemplificar o representar dos categorías distintas: medios tradicionales que proponen realizar la verificación (Fato ou Fake, del portal G1, del Grupo Globo) y medios con origen digital que han propuesto realizar la misma actividad de una manera más independiente 
(Lupa). Inicialmente, según las predicciones de la Organización Mundial de la Salud (OMS) y el Ministerio de Salud de Brasil, el corpus se organizó en función de dos etapas temporales: enero, febrero y marzo - primeros casos de infección y muerte y definición como pandemia; y abril, mayo y junio -aumento y estabilización de casos en el país. La recolección de material ha considerado ambos periodos para cada caso, de modo que se garantice la posibilidad de realizar también análisis comparativos. El objetivo principal es presentar resultados profundos de los estándares de información falsa y, por otro lado, de las estrategias de innovación de la presentación de estas verificaciones de desinformación sobre la COVID-19.

Específicamente, la atención se ha centrado en la frecuencia del enfoque de COVID-19 entre las dos agencias de verificación estudiadas: Fato ou Fake y Lupa. El análisis de framing de la investigación en su conjunto se ha llevado a cabo en dos etapas. En la primera, evaluamos el frame mediático en el proceso de desinformación relacionado con COVID-19. En la segunda, verificamos cómo los materiales de las agencias de verificación confirman o refutan este framing "general"; es decir, identificamos las estrategias de innovación de estas iniciativas periodísticas para combatir la información falsa sobre la pandemia en Brasil. A continuación, presentamos un estudio exploratorio inicial realizado en las agencias de verificación, esencial para los próximos pasos de la investigación general que han realizado los autores.

El Fato ou Fake, un hotsite dentro del portal G1, del Grupo Globo, designó una especie de editorial para las verificaciones acerca de la COVID-19. Aun así, es una página sin estructura propia, cercana a la estructura y estética del formato blog, con las publicaciones ordenadas en orden cronológico inverso y con los artículos más recientes dispuestos a dos columnas. Además de la herramienta de búsqueda, la única forma de navegar es por enlaces, que agregan poco a la navegación (cada uno con tres enlaces y llamados "Verificaciones" y "Cómo llegar").

De enero a junio, la página para evaluar presuntas fake news sobre la pandemia generó 257 publicaciones. De éstas, solo dos no eran exactamente verificaciones, sino un balance de sus producciones. La única publicación de enero, del día 28 , entra en esta categoría. En ésta se informa al público que agregarían todas las publicaciones en su propia página, ya que la publicación solo contiene el enlace en la página precedido por el mensaje "Ver todas las verificaciones relacionadas al coronavirus ". La otra sucedió el último día de abril, cuando dijeron: "Fato ou Fake llega a 150 verificaciones del coronavirus; saber cuáles son los más accedidos". Por lo tanto, se realizaron 255 verificaciones en total, comenzando en febrero de 2020, aunque representaron solo 9. En marzo, cuando la OMS declaró la COVID-19 como una pandemia y cuando se implementaron medidas de aislamiento social en varios estados brasileños, se realizaron un poco menos de dos verificaciones por día, lo que representa un número total de 53. En abril, se alcanzó el pico con casi tres verificaciones por día, llegando a 89, con mucha reducción en los dos meses siguientes, 66 y 38, respectivamente, es decir había poco más de dos por día en mayo y uno por día en junio.

La Agencia Lupa, aunque está vinculada al periódico Folha de S. Paulo, se presenta como un proyecto independiente, que se considera la primera agencia de fact-checking de Brasil. Su página está alojada en el portal UOL, pero, como Fato ou Fake, su estructura de página se asemeja a un blog, con publicaciones en orden cronológico decreciente. En la columna derecha hay enlaces a sus principales redes sociales digitales, explicaciones sobre las colaboraciones establecidas para mantener el sitio, metodología adoptada para la verificación (incluso la organización de las etiquetas) y las formas de contacto, que permiten sugerir verificaciones y nombres de profesionales para comprobar la información recibida. Además, hay enlaces a "Secciones" específicas y al "Archivo" de publicaciones, divididas por año y mes, a partir de octubre de 2015.

De enero a junio, Lupa realizó 298 publicaciones relacionadas con el COVID-19, incluidos temas como el aislamiento social, los tratamientos identificados como soluciones para el virus, las quejas de figuras públicas que romperían la distancia (promoviendo fiestas, por ejemplo), la evidencia de que los ataúdes y los hospitales podrían estar vacíos, entre otras categorías de información. En el estudio exploratorio que detallamos en este artículo, es importante señalar que, de estas publicaciones, 221 fueron verificaciones efectivas, es decir, no consistieron en artículos sobre proyectos especiales y contenido vinculado al proyecto "Lupa na Ciência" y el podcast "Verifica Coronavirus", solo por mencionar dos ejemplos recurrentes entre las publicaciones analizadas.

De enero a junio, se contabilizaron 720 producciones totales en Lupa, de las cuales 298 fueron informaciones sobre la pandemia y 221 fueron verificaciones propiamente dichas. De manera similar Fato ou Fake presentó pocas publicaciones sobre COVID-19 en los meses de enero y febrero, concretamente 17, con 12 verificaciones. En marzo, cuando la OMS declaró la COVID-19 como una pandemia, y cuando se implementaron medidas de aislamiento social en varios estados brasileños, el número aumentó a más del triple en los primeros dos meses del año, llegando a 51 publicaciones y 42 verificaciones. En abril, el salto es significativo, al alcanzarse el pico de publicaciones sobre el coronavirus durante la pandemia: 89 publicaciones en total y 70 verificaciones. En los siguientes dos meses, comienza la disminución, con 74 y 67 publicaciones y, respectivamente, 54 y 43 verificaciones. Aunque en mayo y junio la producción en la pandemia mostró una reducción, Lupa aumenta su total de publicaciones, conside- 


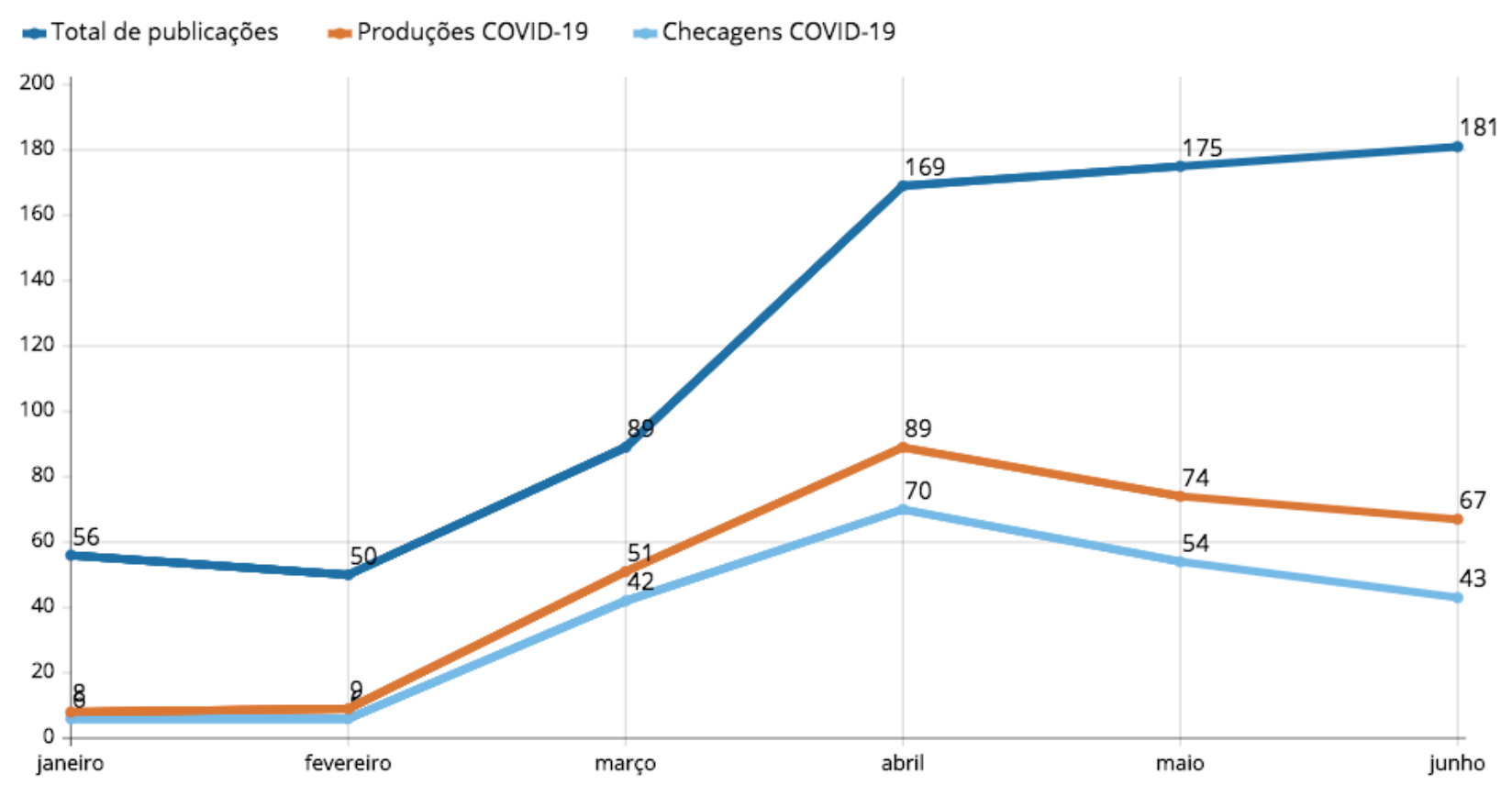

Figura 2. Publicaciones y verificaciones en Lupa entre enero y junio 2020. Elaboración propia.

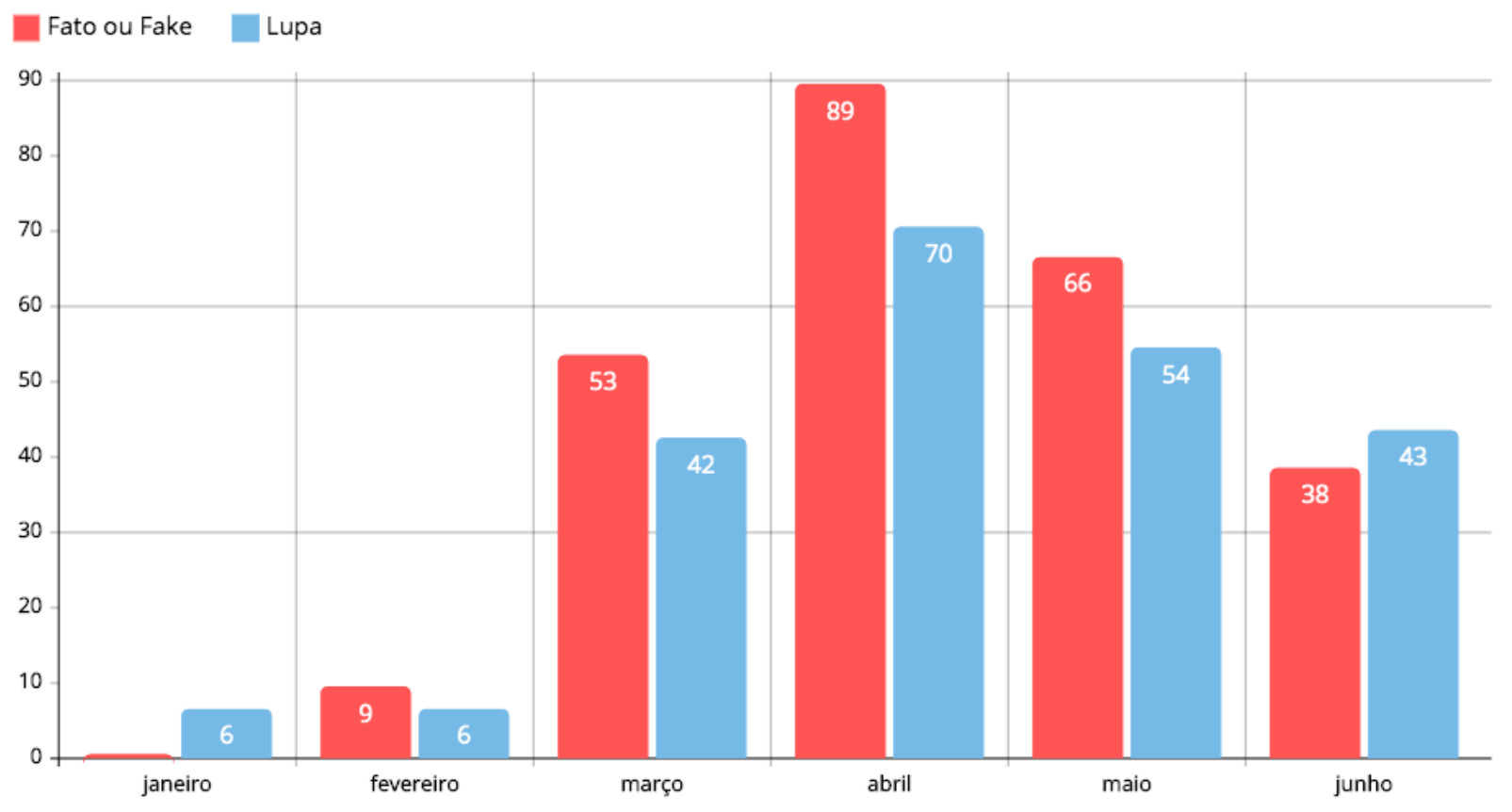

Figura 3. Verificaciones de la COVID-19 en las agencias de enero a junio de 2020. Elaboración propia.

rando también otros temas, comenzando desde 169, en abril, hasta 175 y 181 , en mayo y junio (Figura 2).

En los períodos iniciales de propagación de la enfermedad, en enero y febrero de 2020, las verificaciones en las agencias no alcanzaron el 20\% del material producido. A partir de marzo, sin embargo, las verificaciones se volvieron más hacia la pandemia, con casi dos publicaciones al día. El período coincide con la declaración de COVID-19 como pandemia por parte de la OMG y la implementación de medidas de aislamiento social en varios estados brasileños. En abril, Fato ou Fake produce casi tres verificaciones diarias, cayendo dramáticamente en los próximos dos meses, con poco más de dos y una por día en mayo y junio. En Lupa, el pico no fue tan alto, superando dos verificaciones por día en abril, pero registrando una disminución menos pronunciada en mayo y junio (ver Figura 3). La curva en las dos agencias es similar, aunque más aguda en Fato ou Fake. 


\section{Conclusiones}

La búsqueda de la verdad y la objetividad han de ser los primeros objetivos del periodista leal a su profesión (Silva, 2019). Canavilhas y Ferrari (2018) se refieren a la existencia de una post-verdad, un contexto en el que la verdad adquiere diferentes significados según la interpretación de cada comunidad y sus motivaciones. Este término ha ampliado su uso a raíz de la expresión "política post-verdad", empleada por la revista británica The Economist para referirse a informaciones falsas difundidas en la campaña del Brexit en septiembre de 2016. En suma, las fake news están adquiriendo gran relevancia en los tiempos contemporáneos, ya que generan la posibilidad de que el periodismo utilice el fact-checking para establecerse a través de la credibilidad, "incluso creando nuevos lenguajes para distinguir la verdad de la post-verdad" (Canavilhas; Ferrari, 2018: 39).

Así, parece existir consenso al admitir que las fake news con su proceso de desinformación se proliferan cuando los periódicos disminuyen su credibilidad y exclusividad como componentes de una institución de referencia para la producción y circulación de información (Eichler; Kalsing; Gruszynski, 2018; Silva; Melo 2020; Spinelli; Santos, 2018; Orbegozo, Morales y Larrondo, 2020). Los productores y propagadores de fake news, casi paradójicamente, confían en esta institución para que adquieran más credibilidad o, al menos, disminuyan la de los profesionales. Para garantizar que la mentira sea confiable, el contenido está en línea con la creencia y los valores de la mayoría de los consumidores (Gruszynski et al., 2020; Teixeira; Costa, 2020; Purple; Melo, 2018). Precisamente, la difusión a menudo proviene de figuras de confianza, como amigos y familiares, y llegan solo a quienes ya están de acuerdo, teniendo en cuenta los filtros de las redes sociales.

Incluso en vista del corpus seleccionado para el análisis, es evidente que, a pesar del uso generalizado de las redes sociales para la desinformación, los medios tradicionales siguen siendo las principales fuentes de noticias circuladas en Internet. Esto revela que las tareas de selección y edición de contenido circulado siguen siendo de importancia central en el contexto contemporáneo, especialmente a través de portales en línea de verificación de información. En otras palabras, el trabajo realizado por los periodistas sigue siendo relevante. Sin embargo, esto no significa que estos profesionales necesiten producir más de lo mismo.

La innovación ha sido cada vez más demandada y necesaria. La comprensión de la arquitectura de la información, de la relación de las personas con los medios emergentes y de los nuevos modelos de negocio son algunos de los aspectos a considerar (Machado Filho; Ferreira, 2012). Por lo tanto, es importante notar la diferencia en la navegación que las agencias han dedicado a las verificaciones de COVID-19. Mientras que Fato ou Fake organizó las producciones de manera des- tacada en la página principal, pero con una navegabilidad con pocas opciones, Lupa permite la navegación por categorías debidamente explicadas en la página de inicio de la agencia de fact-checking.

Estos resultados confirman las tendencias globales de las agencias de verificación, cuya presentación principal suele utilizar etiquetas, y su contenido suele tener enlaces para prueba de verificación e imágenes, infografías y videos para facilitar la comprensión de los consumidores (Nyhan; Reifler, 2012; Fernandes; Oliveira; Gomes, 2019). Pero, aunque las etiquetas tienen un uso común en las agencias de verificación, sus elecciones pueden ser subjetivas y crear distorsiones incluso sobre lo que sería una mentira o no (y las gradaciones incluidas).

Aunque las agencias casi nunca verifican las mismas afirmaciones, ya que existe cierto entendimiento de que el trabajo es evaluar lo que aún está en duda, cuando existe esta coincidencia, las etiquetas arrojan resultados diferentes. Este tipo de desacuerdos, a veces discrepantes, por las subjetividades en la catalogación, hace que Santos y Maurer (2020, p.12) afirmen que "la transparencia de los métodos tiene el potencial de traer mayores aportes al público que la clasificación de la información".

Algunos autores (Santos; Maurer, 2020) creen que las agencias hacen solo la primera etapa de un proceso periodístico, la investigación, pero esta afirmación no es del todo correcta. Es cierto que el énfasis comenzó en esta etapa de la rutina periodística, sin embargo, cada vez más, las agencias se preocupan por la presentación del contenido verificado, no solo por motivos comerciales, logrando mayor circulación e ingresos, sino también para que la mentira queda expuesta al mayor número de personas posible, eliminando su aspecto nocivo. En otras palabras, los profesionales de las agencias de verificación escudriñan -especialmente datos-, producen -en relación con enlaces y multimedia- y editan -especialmente en la definición de las etiquetas y la distribución del contenido- su material en detalle. Aunque producen y editan el contenido, con el fin de llegar a una audiencia más amplia y explicar las complejidades de esa información falsa del proceso de desinformación, el papel principal de estas agencias sigue siendo una investigación rigurosa. Sus consumidores realmente quieren saber si esa información es verdadera o no, aunque la mayoría de las agencias hacen más compleja la presentación de este contenido, con etiquetas que no siempre aseguran la completa veracidad o falsedad del contenido.

Este panorama destaca, por tanto, la necesidad de que las agencias no sean soluciones universales o definitivas para combatir la desinformación. Después de todo, cuando se repite una noticia falsa, incluso para negarla, el efecto puede ser el contrario, especialmente si la persona aún no conocía la información incorrecta. Una mejor manera de aumentar la efectividad de la corrección es presentar la poca relevancia de 
la información errónea, especialmente cuando se usa información visual, como imágenes y gráficos. Además, el periodismo no debe dejar espacio a fuentes mentirosas que abogan contra los consensos científicos, en busca de una supuesta y vacía pluralidad, como quienes apoyan el movimiento antivacunas de COVID-19, por ejemplo.

\section{Referencias}

Alencar, M., y Dourado, J. (2020). Fact-checking: checagens da Folha e do Estadão na construção da credibilidade jornalística na internet. Revista Eptic, 22(2), 23-37. https://seer.ufs.br/index.php/eptic/ article/view/11862

Barbeiro, H., y Lima, P. (2002). Manual de telejornalismo: os segredos da notícia na TV. Campus.

Bardin, L. (2004). Análise de conteúdo. Edições 70.

Borcezi, D., y Morais, C. (2019). Fact-checking e a circulação da notícia política: o discurso sobre o fim da Cracolândia. Revista ĺcone, 17(1), 72-84. https://doi.org/10.34176/icone.v17i1.237623

Briggs, M. (2013). Journalism next: a practical guide to digital reporting and publishing. $2^{a}$ Ed. SAGE.

Canavilhas, J., y Ferrari, P. (2019). Fact-checking: o jornalismo regressa às origens, en Schroeder-Buitoni, D. (org.) Jornalismo em tempo de transformaçâo: desafios de produção e de ação (pp. 30-49). Editora Sulina.

Canavilhas, J., y Satuf, I. (2014). Jornalismo em transição, en Fidalgo, A., y Canavilhas, J. (Org.). Comunicação digital: 10 anos de investigação (pp. 33-58). Livros Labcom.

Charaudeau, P. (2009). Discurso das mídias. Contexto.

Eichler, V., Kalsing, J., y Gruszynski, A. (2018). 0 ethos do jornal 0 Globo e a campanha contra as fake news. Media \& Jornalismo, 18(32), 139-154. https://doi.org/10.14195/2183-5462_32_10

Entman, R. (1993). Framing: Toward clarification of a fractured paradigm. Journal of Communication, 43(4), 51-58. https://doi org/10.1111/j.1460-2466.1993.tb01304.x

Fernandes, C., Oliveira, L., y Gomes, V. (11-14 de junio de 2019). Tensionamentos entre campos sociais: as fake news e a reconfiguração do campo comunicacional e político na era da pós-verdade. Anais do XXVIII Encontro Anual da Compós. PUC-RS, Porto Alegre.

Graves L., y Cherubini F. (2016). The rise of fact-checking sites in Europe. Reuters Institute for the Study of Journalism. https:// reutersinstitute.politics.ox.ac.uk/sites/default/files/research/ files/The\%2520Rise\%2520of\%2520Fact-Checking\%2520Sites\%2520in\%2520Europe.pdf.

Gruszynski, A., Kalsing, J., Rizzo-Hoewell, G., y Gandon-Brandão, C. (2020). Fact-checking e saúde: análise da seção 'Verdade ou Boato' de GaúchaZH. Revista Eletrônica de Comunicação, Informação \& Informação em Saúde, 14(1), 51-71. https://doi.org/10.29397/reciis. v14i1.1860

Herreros, M. C. (2003). Información Televisiva. Sintesis.

Jenkins, H. (2014). Cultura da conexão. Aleph.

Jenkins, H. (2009). Cultura da Convergência. 2. ed. Aleph.

Lage, N. (2009). A reportagem: teoria e técnica de entrevista e pesquisa jornalística. Record.
Larrondo, A. (2007). The Challenge of Online Journalistic Language to Narrative Forms. ZER english edition, 41-61.

Lemos, A. (2004). Cibercultura e Mobilidade. Revista Razón y Palabra (41), http://www.razonypalabra.org.mx/anteriores/n41/alemos.html

Machado, E. (2003). 0 ciberespaço como fonte para os jornalistas. Anais da BOCC. Universidade da Beira Interior. http://www.bocc.ubi. pt/pag/machado-elias-ciberespaco-jornalistas.pdf

Machado Filho, F., y Ferreira, M. (2012). Jornalismo audiovisual: da tela da TV para outras telas. Brazilian Journalism Research, 8(2), 135-153. https://doi.org/10.25200/BJR.v8n2.2012.421

Meneses, J. P. (2018). Sobre a necessidade de conceptualizar o fenómeno das fake news. Observatorio (OBS*), (Special Issue), 37-53. https://doi.org/10.15847/obsOBS12520181376

Moretzsohn, S. (2002). Jornalismo em tempo real: o fetiche da velocidade. Editora Revan.

Nyhan, B., y Reifler, J. (2012). Misinformation and fact-checking: research findings from Social Science. New America Foundation.

Orbegozo, J., Morales, J., y Larrondo, A. (2020). Desinformación en redes sociales: ¿compartimentos estancos o espacios dialécticos? El caso Luther King, Quim Torra y El Confidencial. Revista Mediterránea de Comunicación, 11(2), 55-69. https://doi.org/10.14198/ MEDCOM2020.11.2.2

Palacios, M., Barbosa, S., da Silva, F.F., y da Cunha, R. (2015). Jornalismo móvel e inovações induzidas por affordances em narrativas para aplicativos em tablets e smartphones, en Canavilhas, J., Satuf, I. (Orgs.). Jornalismo para Dispositivos móveis (pp. 7-42). Livros Labcom.

Quinn, S. (2005). Convergent journalism. Peter Lang Publishing.

Roxo, M. A., y Melo, S. (2018). Hiperjornalismo: uma visada sobre fake news a partir da autoridade jornalística. Famecos, 25(3), ID30572. https://doi.org/10.15448/1980-3729.2018.3.30572

Santos, C., y Maurer, C. (2020). Potencialidades fact-checking no combate à desinformação. Comunicação \& Informação, 23, 1-14. https://doi.org/10.5216/ci.v23i.57839

Schudson, M. (2008). News and democratic society. The Hedgehog Review, 10(2), 7-21.

Schudson, M., y Zelizer, B. (2017). Fake News in Context, en Understanding and Addressing the Disinformation Ecosystem (pp. 1-4). Annenberg School for Communication.

Silva, A. F. (2019). Porque é que as fake news se transformaram em protagonistas do jornalismo contemporâneo? Comunicação Pública, 14(26). https://doi.org/10.4000/cp.4139

Silva, F. (2015). Jornalismo móvel. EDUFBA.

Silva, M; y Melo, S. (2020). Fake news: fronteiras do jornalismo e circulação de (des)informação sobre saúde. Revista Eletrônica de Comunicação, Informação \& Informação em Saúde, 14(1), 1-5. https:// doi.org/10.29397/reciis.v14i1.2047

Sodré, M. (2009). A Narração do Fato: notas para uma teoria do acontecimento. Vozes.

Sousa, J. (2000). As notícias e os seus efeitos. MinervaCoimbra.

Spinelli, E., y Santos, J. (2018). Jornalismo na era da pós-verdadefact-checking como ferramenta de combate às fake news. Revista Observatório, 4(3), 759-782. https://doi.org/10.20873/uft.24474266.2018v4n3p759 
Stovall, J. (2004). Web journalism. Pearson Education.

Tandoc Jr., E., Lim, Z. W., y Ling, R. (2018). Defining "Fake News": a typology of scholarly definitions. Digital Journalism, 6(2), 137-153. https://doi.org/10.1080/21670811.2017.1360143

Traquina, N. (2005). Teorias do Jornalismo: porque as notícias são como são. Vol I. Insular, 2a ed.

Träsel, M., Lisboa, S., y Reis, G. (5-8 de junio de 2018). Indicadores de credibilidade no Jornalismo. XXVII Encontro Anual da Compós. PUC-MG. Belo Horizonte - MG.

Tuchmann, G. (1978). Making News. The Free Press.

Virissimo, V. A. (2009). Apuração na Internet. [Dissertação de mestrado em Jornalismo, Universidade Federal de Santa Catarina]. http://repositorio.ufsc.br/xmlui/handle/123456789/93028

Ward, M. (2006). Jornalismo online. Roca.

Wolf, M. (2005). Teorias das comunicações de massa. $2^{\text {a }}$ ed. Martins Fontes.

Zago, G. (2010). Circulação jornalística no Twitter: apontamentos para discussão. Anais do VII Encontro Nacional de Pesquisadores em Jornalismo (pp. 1-14). SBPJOR.

Zago, G. (2011). Recirculação jornalística no Twitter: motivações dos interagentes para filtrar e comentar notícias. Lumina, 5(2), 1-16. https://periodicos.ufjf.br/index.php/lumina/article/view/21010

\section{CV}

Allysson Martins. Profesor de Periodismo y coordinadora del MíDI - Grupo de Investigación en Medios Digitales e Internet de la Universidad Federal de Rondônia (UNIR). Doctorado en Comunicación y Cultura Contemporáneas por la Universidad Federal de Bahía (UFBA).

Juliana Teixeira. Doctora en Comunicación y Cultura Contemporánea por la Universidad Federal de Bahía (Salvador / Brasil) y en Ciencias de la Comunicación por la Universidad de Beira Interior (Covilhã / Portugal), a través del régimen de co-tutela entre las dos instituciones. Entre 2016 y 2018, realizó posdoctorado en la Universidad Federal de Piauí, donde actualmente es profesora del Departamento de Comunicación Social y del Programa de Posgrado en Comunicación Social. Es líder del grupo de investigación Periodismo, Innovación e Igualdad (JOII - www.joiiufpi.com.br).

Ainara Larrondo. Doctora en Periodismo, Máster en Historia Contemporánea. Profesora Titular de la Universidad del País Vasco. Materias de docencia e investigación: Redacción y Reportajes, Periodismo Online, Innovación en Comunicación, Comunicación Organizacional, Comunicación Política, Género, Medios y Comunicación. Investigadora Principal del Grupo de Investigación Consolidado 'Gureiker' (IT1112-16).

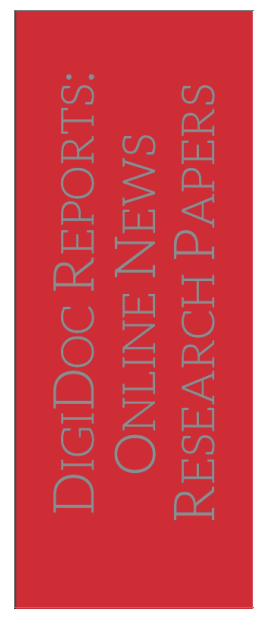

\section{Researching the news \\ in the hybrid media system: An expert panel report}

Irene Costera Meijer, Richard Rogers,

Oscar Westlund \& Tamara Witschge

Javier Díaz-Noci \& Ana Serrano (eds.)

\section{ocm Observatorio deCibermedios}

\section{https://observatoriocibermedios.upf.edu/}

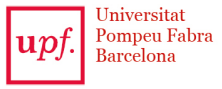

Departamento de Comunicación Grupo DigiDoc

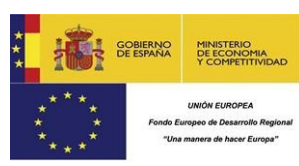

El Observatorio de Cibermedios (OCM) forma parte del proyecto del Plan Nacional "Narración interactiva y visibilidad digital en el documental interactivo y el periodismo estructurado". RTI2018-095714-B-C21 (MINECO/FEDER), Ministerio de Ciencia, Innovación y Universidades (España). 